\title{
Caractérisation et typologie des systèmes d'élevage de la poule locale du Burkina Faso
}

\author{
S. Pindé ${ }^{1,2,3 *}$, A.S.R. Tapsoba ${ }^{1}$, F. Traoré ${ }^{1}$, R. Ouédraogo ${ }^{1}$, S. Ba $^{1}$, M. Sanou${ }^{1}$, A. Traoré ${ }^{1}$ H.H. \\ Tamboura ${ }^{1}$, J. Simporé ${ }^{3}$ \\ 'Laboratoire de Biologie et Santé animales (LaBioSA), Institut de l'Environnement et de Recherches Agricoles (INERA), 04 \\ BP 8645 Onagadougou 04, Burkina Faso \\ ${ }_{2}^{2}$ Ministère des Ressources Animales et Halieutiques (MRAH), 01 BP: 7026 Onagadougou 01, Burkina Faso \\ ${ }^{3}$ Laboratoire de Biologie Moléculaire et de Génétique (LABIOGENE), Université Joseph KI-ZERBO (UJKZ), 03 BP 7021 \\ Onagadougou 03, Burkina Faso \\ Auteur correspondant: Dr Amadou Traoré, DVM, PhD, Directeur de Recherches (CAMES)Laboratoire de Biologie et santé \\ animales, INERA/CNRST, Tél: (00226) 7042 94 40, (00226) 788084 10,Email : traore pa@,yahoo.fr
}

Mots clés : Systèmes d'élevage, poules locales, zones agro-écologiques, Burkina Faso

Keywords : Farming systems, local chicken, agro-ecological zones, Burkina Faso

Publication date 30/11/2020, http://m.elewa.org/Journals/about-japs /

\section{RÉSUMÉ}

La présente étude est une contribution à l'établissement des caractéristiques et de la typologie des systèmes d'élevage de la poule locale dans les 3 zones agro écologique du Burkina Faso. Des informations ont été collectées sur 251 éleveurs de poules locales à travers une enquête de type transversale dans les 3 zones agro-écologiques, du Burkina Faso. Les résultats indiquent que l'élevage des poules locales est pratiqué principalement par des autochtones $(84,91 \%)$ et des hommes $(70,26 \%)$ dans un système extensif $(63,83 \%)$. Les enquêtés sont pour la plupart des adultes $(66,38 \%)$, sont mariés $(92,41 \%)$, n’ont reçu aucune formation technique $(45,69 \%)$ et pratiquent l'agriculture comme activité principale $(67,93 \%)$. Les petits éleveurs de poules locales (effectif inférieur ou égal à 25 têtes) sont les plus nombreux (40,56\%). Les enquêtés disposent majoritairement de poulaillers/abris (76,37\%), utilisent des mangeoires $(58,65 \%)$, des abreuvoirs $(73 \%)$ et distribuent principalement des céréales à leurs poules. Les maladies aviaires sont la première cause de mortalités dans les effectifs visités $(89,22 \%)$, malgré que presque la quasi-totalité des éleveurs $(98,77 \%)$ administrent des traitements sanitaires à leurs poules. L'âge de la maturité sexuelle chez les poules mâles est en moyenne de 5,46 $\pm 0,12$ mois et de 5,49 $\pm 0,10$ mois chez les femelles, avec une différence statistiquement significative pour ce paramètre, au seuil de $\mathrm{p}<0,05$, entre les femelles des 3 zones agroécologiques. Le nombre d'œufs pondus par cycle et par poule dans la zone d'étude est en moyenne de 11,52 $\pm 0,17$ œufs, avec la zone sahélienne qui a le nombre moyen d'œufs pondus le plus élevés $(12,6 \pm 0,32$ œufs). La majorité des enquêtés $(75,58 \%)$ affirme que la période de ponte favorable dans leur exploitation s'étale du début saison des pluies à la fin de la saison sèche froide. L'âge de la réforme des poules dans la zone d'étude est, en moyenne, de $36,82 \pm 1,63$ mois pour les femelles et de 24,55 $\pm 1,16$ mois chez les mâles avec une forte variabilité existant au niveau surtout des femelles des différentes zones agro-écologiques. L'âge moyen à la réforme des femelles $(36,82 \pm 1,63$ mois) révèle une différence statistiquement significative, à $\mathrm{p}<0,05$, entre les oiseaux de la zone sahélienne et ceux des zones soudanienne et soudano-sahélienne. 


\begin{abstract}
This study is a contribution to the establishment of the characteristics and the typology of the farming systems of the local hen in the 3 agro-ecological zones of Burkina Faso. Information was collected on 251 local chicken farmers through a cross-sectional survey in the 3 agroecological zones of Burkina Faso. The results indicate that the rearing of local chicken is practiced mainly by natives $(84.91 \%)$ and men $(70.26 \%)$ in an extensive system $(63.83 \%)$. Respondents are mostly adults $(66.38 \%)$, are married $(92.41 \%)$, have received no technical training $(45.69 \%)$ and practice agriculture as their main activity $(67.93 \%)$. The smallest local chicken breeders (number less than or equal to 25 heads) are the most numerous (40.56\%). Respondents have chicken coops / shelters (76.37\%), use feeders $(58.65 \%)$, drinkers $(73 \%)$ and mainly distribute cereals to their hens. Avian diseases are the leading cause of mortality in the numbers visited (89.22\%), although almost almost all breeders $(\mathbf{9 8 . 7 7 \% )}$ ) administer sanitary treatments to their hens. The age of sexual maturity in male hens is on average 5.46 \pm 0.12 months and $5.49 \pm 0.10$ months in females, with a statistically different significant for this parameter, at the threshold of $\mathrm{p}<0.05$, between the females of the 3 agro-ecological zones. The number of eggs laid per cycle and per hen in the study area averages $11.52 \pm 0.17$ eggs, with the Sahelian zone having the highest average number of eggs laid (12.6 $\pm 0.32 \mathrm{eggs})$. The majority of respondents $(\mathbf{7 5 . 5 8} \%)$ say that the favorable spawning period in their exploitation extends from the beginning of the rainy season to the end of the cold dry season. The age of hen reform in the study area is, on average, 36.82 \pm 1.63 months for females and $24.55 \pm 1.16$ months in males with high variability. level especially of females from different agroecological zones. The culling age of females (36.82 \pm 1.63 months) reveals a statistically significant difference, at $\mathrm{p}<0.05$, between birds in the Sahelian zone and those in the Sudanian and Sudano-Sahelian zones.
\end{abstract}

\section{INTRODUCTION}

Au Burkina Faso, à l'instar des pays en développement, l'élevage de la volaille revêt une importance significative en milieu rural et représente la première source d'approvisionnement des populations en protéines animales (Zaman et al., 2004). Il constitue la principale source de revenus des ménages pauvres en milieu rural et joue un important rôle culturel (MRA/PNDEL, 2010). La filière avicole burkinabè compte un effectif d'environ 44 millions de têtes et est subdivisée en deux secteurs : un secteur traditionnel particulièrement actif ( $90 \%$ du cheptel) dont les producteurs sont les paysans et un secteur moderne (MRAH/DGESS, 2018). Le développement et la rentabilité de cette filière sont intimement liés à la maîtrise des pathologies et à celle de l'alimentation qui sont à leur tour tributaires des systèmes d'élevage (Cothenet et Bastianelli, 1999). Selon Ouattara (2015), 3 systèmes de production avicole coexistent au
Burkina Faso. Il s'agit (i) du système extensif ou aviculture traditionnelle pure, (ii) du système semi-intensif ou aviculture traditionnelle améliorée et (iii) du système intensif ou aviculture moderne dans lequel des races exotiques sont élevées en claustration totale. Il est important de noter cependant que les informations sur les 2 premiers systèmes sont d'ordre global et disparates. Au niveau de la volaille, seule la pintade a bénéficié récemment (Traoré, 2018) d'une attention particulière, en termes de typologie des systèmes d'élevage selon les zones agro écologiques. Cette insuffisance au niveau de la poule locale du Burkina, induit une faible connaissance des habitudes d'élevage, des catégories d'acteurs qui s'adonnent à l'élevage de poules locales et donc une faible maîtrise des performances zootechniques et des contraintes liées à sa production en rapport avec les différentes zones agro écologiques. Toute chose pouvant impacter négativement sur 
l'exploitation rationnelle et optimale de cette espèce et la mise en place de programmes de développement avicoles adaptés aux différentes régions du Burkina Faso. La présente étude est une contribution à l'établissement des caractéristiques et de la typologie des systèmes d'élevage de la poule locale dans les 3 zones agro écologique du Burkina Faso.

\section{MATÉRIEL ET MÉTHODES}

\subsection{Site d'échantillonnage: La présente}

étude a été réalisée au Burkina Faso entre juillet 2018 et janvier 2019. Elle a couvert 251 exploitation avicoles dans 06 régions (Centre, Centre-Est, Centre-Nord, Plateau Central, Hauts-Bassins et Sahel). Dans chaque région, une (01) province a été retenue. Sur les 06 provinces, 11 communes regroupant 57 villages ont été concernés par les enquêtes. Les sites d'échantillonnage couvrant les 3 zones agroécologiques du pays sont présentés dans la Figure 1.

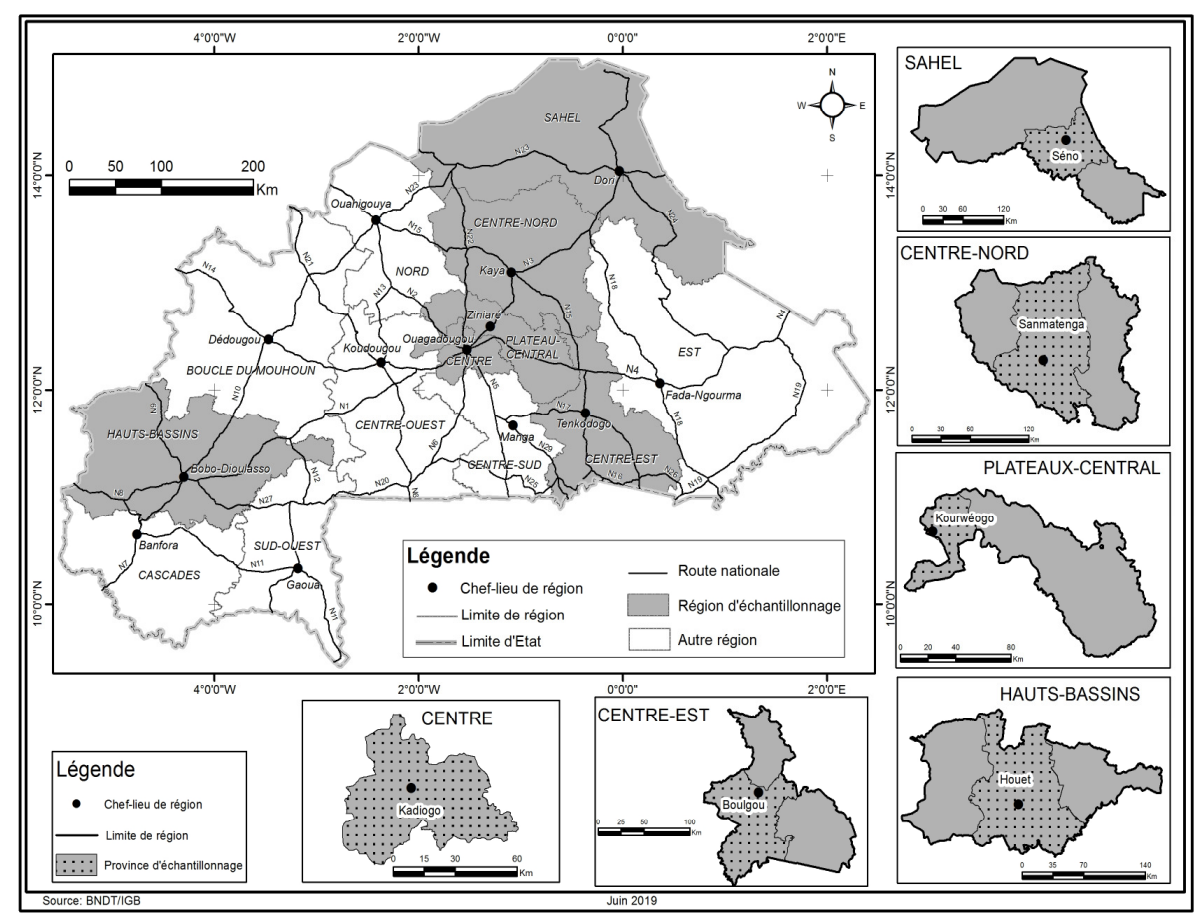

Figure 1: Carte de localisation de la zone d'étude (IGB, 2019).

\subsection{Critères de choix des fermes} avicoles: L'étude a exclusivement porté sur l'aviculture traditionnelle. Le choix des éleveurs pour les enquêtes s'est fait sur la base (i) de l'effectif de leur cheptel qui devrait comporter un minimum de 3 individus en âge de se reproduire et (ii) de l'éloignement suffisant des élevages afin de limiter l'apparentement entre les animaux échantillonnés (au moins $1 \mathrm{~km}$ entre les exploitations). Les éleveurs de poules ont été sélectionnés avec l'appui des services techniques déconcentrés du Ministère des ressources animales et halieutiques dans leurs zones respectives. Suivant ces critères, 10 à 15 éleveurs ont été sélectionnés par les chefs de Zone d'Appui Technique en Elevage (ZATE) de chaque commune. Chaque éleveur a fait l'objet d'une interview suivant un questionnaire.

3.3 Enquêtes socio-économiques et zootechniques: Une enquête de type transversal a été menée et a permis de collecter des informations sur l'élevage, le système avicole, les infrastructures et équipements, les pathologies aviaires, la gestion de la santé des volailles, la production (nombre d'œufs/femelles/an, taux d'éclosion, nombre 
d'œufs/cycle de ponte, le nombre d'œufs mise à couver/femelle/an, nombre de cycle de ponte/an, période de ponte, âge à la maturité sexuelle et âge de réforme) et les contraintes de production. La relation entre le cheptel et les concepts sociaux (âge, religion, sexe) est également enregistrée.

\subsection{Traitement et analyse des données :}

Les données socio-économiques et de production de l'enquête ont été saisies avec le logiciel Excel 2016. Pour ces données, des statistiques descriptives, une analyse des correspondances multiples (MCA) et une analyse de la classification hiérarchique (HCA, algorithme de Ward) ont été réalisées à l'aide du logiciel R ( $\mathrm{R}$ core team, 2019, version 3.6.0) afin d'établir une typologie (package FactoMineR, fonctions MCA et regroupement hiérarchique sur des composants principaux). Les variables utilisées pour le MCA et le HCA sont décrites dans le Tableau 1.

Tableau 1: Variables et modalités utilisées dans l'analyse factorielle des correspondances multiples.

\begin{tabular}{|c|c|c|}
\hline Variables & Codes & Modalités \\
\hline \multirow{3}{*}{$\begin{array}{l}\text { Zone agro- } \\
\text { écologique }\end{array}$} & \multirow{3}{*}{ Zagroeco } & saheli : sahélienne \\
\hline & & soudan. Saheli : soudano-sahélienne \\
\hline & & soudan : soudanienne \\
\hline \multirow{4}{*}{ Ethnie } & \multirow{4}{*}{ Ethnie } & ethnie1 : Mossi \\
\hline & & ethnie2: Peulh \\
\hline & & ethnie3 : Bobo \\
\hline & & $\begin{array}{l}\text { ethnie4 : gourounsi, tiefo, bissa, yarega, koursassi, samo, } \\
\text { gourmantche }\end{array}$ \\
\hline \multirow{2}{*}{$\begin{array}{l}\text { Motivation du } \\
\text { producteur }\end{array}$} & \multirow{2}{*}{ Objectifs } & objet1 : revenu \\
\hline & & objet 2 : rites et prestiges \\
\hline \multirow{2}{*}{ Système d'élevage } & \multirow{2}{*}{ Syst } & syst1: système semi-intensif \\
\hline & & syst 2 : système extensif \\
\hline \multirow{2}{*}{$\begin{array}{l}\text { Cause de } \\
\text { mortalité }\end{array}$} & \multirow{2}{*}{ cause mortalité } & mort1: maladie \\
\hline & & mort2: prédateurs \\
\hline \multirow{3}{*}{$\begin{array}{l}\text { Effectifs des } \\
\text { élevages }\end{array}$} & \multirow{3}{*}{ Effectif } & effect1: $\leq 25$ têtes \\
\hline & & effect $2:$ entre $[26 ; 50[$ têtes \\
\hline & & effect3 $: \geq 50$ têtes \\
\hline \multirow{5}{*}{$\begin{array}{l}\text { Niveau } \\
\text { d'éducation }\end{array}$} & \multirow{5}{*}{ Educat } & educat0 : ne sais ni lire, ni écrire \\
\hline & & educat1 : alphabétisé y compris l'école coranique \\
\hline & & educat 2 : primaire \\
\hline & & educat 3 : secondaire \\
\hline & & educat 4 : supérieur \\
\hline \multirow{2}{*}{$\begin{array}{l}\text { Origine de de } \\
\text { l'éleveur }\end{array}$} & \multirow{2}{*}{ Origin } & origine1 : autochtone \\
\hline & & origine $2:$ allogène \\
\hline \multirow{2}{*}{$\begin{array}{l}\text { Expérience de } \\
\text { l'éleveur }\end{array}$} & \multirow{2}{*}{ Exp } & expjeun : petite expérience $\leq 5$ ans \\
\hline & & expanc : grande expérience $\geq$ 6ans \\
\hline \multirow{3}{*}{$\begin{array}{l}\text { Niveau de } \\
\text { formation }\end{array}$} & \multirow{3}{*}{ Formati } & format $0:$ aucune formation \\
\hline & & formatavi : formation en aviculture \\
\hline & & formatelev : autres formation en élevage \\
\hline \multirow{2}{*}{$\begin{array}{l}\text { Sexe du } \\
\text { producteur }\end{array}$} & \multirow{2}{*}{ Sexe } & hom : masculin \\
\hline & & fem : féminin \\
\hline \multirow{3}{*}{$\begin{array}{l}\text { Age du } \\
\text { producteur }\end{array}$} & \multirow{3}{*}{ Agep } & jeune $: \leq 36$ ans \\
\hline & & adult : [37;60[ ans \\
\hline & & vieux $: \geq 60$ ans \\
\hline
\end{tabular}


Les librairies lsmeans et hglm ont été également utilisées en vue de l'estimation des moyennes des

\section{RÉSULTATS}

4.1 Caractéristiques socio-économiques des éleveurs : La présente étude a montré que l'élevage des poules locales est une activité majoritairement masculine $(70,26 \%)$ et l'objectif principal visé (motivation) pour cette activité est la recherche de revenus $(97,84 \%)$. Les éleveurs de poules locales ont pour activité principale l'agriculture, $67,93 \%$ contre $27,43 \%$ qui ont pour moindres carrées pour les données de production.

activité principale l'élevage et $2,11 \%$ le commerce. Plus de la moitié des enquêtés (51,69\%) n'appartient pas à une organisation professionnelle agricole (OPA) et compte une forte proportion d'illettrés $(40,95 \%)$. Ils sont diversement instruits et n'ont reçu aucune formation en élevage tels que présentés dans le Tableau 2.

Tableau 2: Caractéristiques socio-économiques des éleveurs de poules.

\begin{tabular}{|c|c|c|c|c|}
\hline \multirow[b]{2}{*}{ Paramètres } & \multicolumn{4}{|c|}{ Zones Agro-écologiques } \\
\hline & Soudanienne & Soudano-Sahélienne & Sahélienne & Général \\
\hline \multicolumn{5}{|l|}{ Sexe $(\%)$} \\
\hline Féminin & 17,07 & 27,66 & 46 & 29,74 \\
\hline Masculin & 82,93 & 72,34 & 54 & 70,26 \\
\hline \multicolumn{5}{|l|}{ Age (\%) } \\
\hline Jeune ( $\leq 36$ ans) & 9,76 & 26,24 & 22 & 22,41 \\
\hline Adulte [37 et 59 ans] & 75,61 & 63,83 & 66 & 66,38 \\
\hline Vieux ( $\geq 60$ ans) & 14,63 & 9,93 & 12 & 11,21 \\
\hline \multicolumn{5}{|l|}{ Situation matrimoniale (\%) } \\
\hline Célibataire & 2,17 & 4,96 & 2 & 3,80 \\
\hline Marié & 91,30 & 92,20 & 94 & 92,41 \\
\hline Divorcé & 2,17 & 0,71 & - & 0,84 \\
\hline Veuf (ve) & 4,35 & 2,13 & 4 & 2,95 \\
\hline \multicolumn{5}{|l|}{ Formation en élevage $(\%)$} \\
\hline Aucune & 63,41 & 41,84 & 42 & 45,69 \\
\hline Aviculture & 36,59 & 46,10 & 32 & 41,38 \\
\hline Autres (embouche, lait, santé) & - & 12,06 & 26 & 12,93 \\
\hline \multicolumn{5}{|l|}{ Activité principale $(\%)$} \\
\hline Agriculture & 63,04 & 70,21 & 66 & 67,93 \\
\hline Commerce & - & 2,84 & 2 & 2,11 \\
\hline Elevage & 34,78 & 24,11 & 30 & 27,43 \\
\hline Elève & - & 1,42 & - & 0,84 \\
\hline Fonctionnaire & - & 0,71 & - & 0,42 \\
\hline Artisan & - & - & 2 & 0,42 \\
\hline Salarié du privé & 2,17 & - & - & 0,42 \\
\hline Pasteur & - & 0,71 & - & 0,42 \\
\hline
\end{tabular}

Il ressort également que l'élevage des poules locales est pratiqué par $84,91 \%$ d'autochtones contre 15,09\% d'allogènes (Figure 2a) ; ceci avec les ethnies mossi $(40,95 \%)$ et peulh $(34,91 \%)$ qui sont majoritaires, contre $24,14 \%$ constitué de bobo, gourounsi, tiefo, bissa, yarega, koursassi, samo, gourmantché (Figure 2b). 


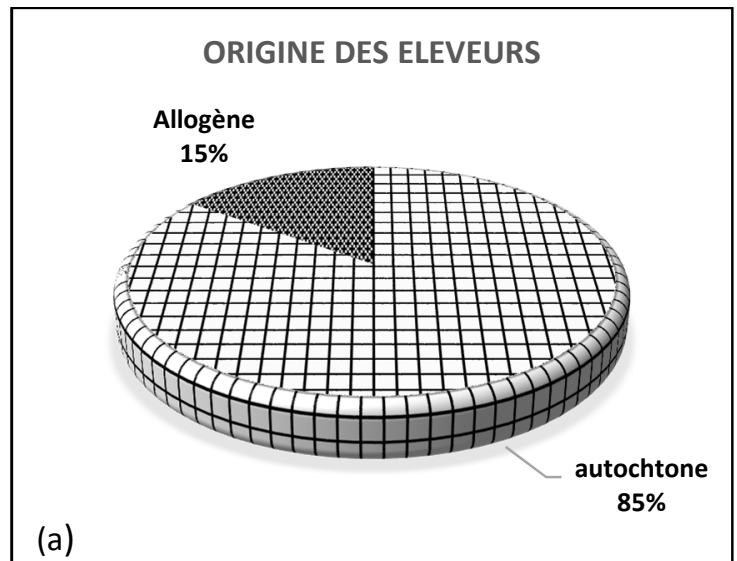

Figure 2: Origine et ethnie des éleveurs

4.2 Conduite de l'élevage : Les données indiquent que ce sont les petits éleveurs de poules locales (effectifs inférieurs à 25 têtes) qui sont les plus nombreux (40,56\%), suivi des éleveurs moyens (effectifs compris entre 26 et 50 têtes) dans la zone d'étude. Les zones soudanienne et soudano-sahélienne présentent les taux les plus élevés de grands éleveurs avec respectivement $26,83 \%$ et $25,53 \%$ des enquêtés qui ont des effectifs pouvant atteindre 2000 têtes dans certaines régions telles que celle du Centre. La majorité $(76,37 \%)$ des éleveurs de poules locales de la zone d'étude dispose de poulaillers/abris construits en banco et souvent de petites dimensions. Les poulaillers de notre zone d'étude disposaient pour la plupart d'équipements d'élevage (abreuvoirs : 58,65\% et mangeoires: 73\%). Dans toutes les exploitations visitées, les poules trouvent l'essentiel de leur alimentation dans la nature. Un apport supplémentaire en céréale (principalement le maïs, sorgho ou petit mil) est, ensuite, généralement fait par l'éleveur 1 à 4 fois dans la journée. Seulement 14,35\% des éleveurs utilisent l'aliment volaille (aliments composés), produits par les unités de fabrique d'aliments. Presque tous les éleveurs $(98,77 \%$ ) de la zone d'étude administrent des traitements sanitaires à leurs poules. Ils utilisent majoritairement la médecine moderne conventionnelle, contre une faible proportion $(3,36 \%)$ qui ont recours à la médecine traditionnelle. Cependant, il ressort néanmoins que ce sont les maladies, notamment la pseudo-peste aviaire couramment appelée maladie de Newcastle, qui constitue la principale cause de mortalités $(89,22 \%)$ dans la zone d'étude.

4.3 Performances de production et de reproduction: Le Tableau 3 présente quelques paramètres de productions dans les 3 zones agro-écologiques. Il ressort en effet que l'âge moyen d'entrée en ponte dans la zone d'étude est de 6,57 $\pm 0,10$ mois. Il existe une différence statistiquement significative pour ce paramètre $(p<0,05)$, entre les femelles des 3 zones agroécologiques. La durée de cette période diminue de la zone soudanienne $(7,45 \pm 0,35$ mois chez les

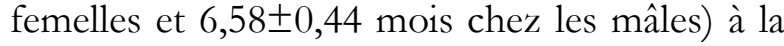
zone sahélienne $(5,00 \pm 0,17$ mois chez les femelles et 4,69 $\pm 0,22$ mois chez les femelle) en passant par la zone soudano-sahélienne $(5,71 \pm 0,14$ mois chez les femelles et $5,51 \pm 0,12$ mois chez les mâles). Le nombre de cycles de ponte par poule et par an qui est en moyenne de

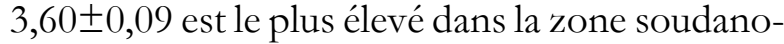
sahélienne $(3,85 \pm 0,12)$. Le nombre de cycles de ponte par an des poules des zones soudanienne et sahélienne diffère significativement de celui de la zone soudano-sahélienne $(\mathrm{p}<0,05)$. Le nombre d'œufs pondus par femelle et par an est

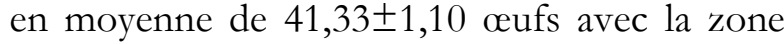
soudano-sahélienne qui présente la meilleure performance $(42,00 \pm 1,44$ œufs), suivie de la zone sahélienne (40,50 $\pm 2,15$ œufs) et de la zone

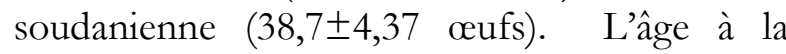
réforme des poules dans la zone d'étude est, en moyenne, plus élevé chez les femelles (36,82 $\pm 1,63$ mois) que chez les mâles 
(24,55 $\pm 1,16$ mois). Une forte variabilité existe au niveau surtout des femelles des différentes zones agro-écologiques avec la zone sahélienne dont les femelles sont réformées tardivement, soit autour de plus de 4 ans en moyenne $(51,7 \pm 2,81$ mois).

Tableau 3: Performances de ponte et de reproduction des poules locales de la zone d'étude.

\begin{tabular}{l|l|l|l|l}
\hline \multirow{2}{*}{ Paramètres } & \multicolumn{4}{|c}{ Populations de poules } \\
\cline { 2 - 5 } & Soudanienne & Soudano-sahélienne & Sahélienne & Général \\
\hline Age maturité sexuelle femelle (mois) & $7,45 \pm 0,35^{\mathrm{a}}$ & $5,51 \pm 0,12^{\mathrm{b}}$ & $5,00 \pm 0,17^{\mathrm{c}}$ & $5,49 \pm 0,10$ \\
Age maturité sexuelle mâle (mois) & $6,58 \pm 0,44^{\mathrm{a}}$ & $5,71 \pm 0,14^{\mathrm{a}}$ & $4,69 \pm 0,22^{\mathrm{b}}$ & $5,46 \pm 0,12$ \\
Age entrée ponte (mois) & $7,41 \pm 0,38^{\mathrm{a}}$ & $6,66 \pm 0,13^{\mathrm{a}}$ & $6,16 \pm 0,19^{\mathrm{a}, \mathrm{b}}$ & $6,57 \pm 0,10$ \\
Nombre œufs/femelle/an & $38,7 \pm 4,37^{\mathrm{a}}$ & $42,00 \pm 1,44^{\mathrm{a}}$ & $40,50 \pm 2,15^{\mathrm{a}}$ & $41,33 \pm 1,10$ \\
Taux d'éclosion & $85,9 \pm 4,00^{\mathrm{a}}$ & $75,6 \pm 1,31^{\mathrm{b}}$ & $77,4 \pm 1,197^{\mathrm{b}}$ & $76,90 \pm 1,02$ \\
Nombre œufs/cycle & $11,9 \pm 0,65^{\mathrm{a}}$ & $11,00 \pm 0,21^{\mathrm{a}}$ & $12,6 \pm 0,32^{\mathrm{a}}$ & $11,52 \pm 0,17$ \\
Eufs mis à couvé/femelle/an & $38,6 \pm 4,49^{\mathrm{a}}$ & $37,4 \pm 1,48^{\mathrm{a}}$ & $39,7 \pm 2,21^{\mathrm{a}}$ & $38,08 \pm 1,13$ \\
Nombre cycle/an & $3,19 \pm 0,37^{\mathrm{a}}$ & $3,85 \pm 0,12^{\mathrm{b}}$ & $3,21 \pm 0,18^{\mathrm{a}}$ & $3,60 \pm 0,09$ \\
Age reforme femelle (mois) & $38,80 \pm 5,7^{\mathrm{a}}$ & $30,00 \pm 1,87^{\mathrm{a}}$ & $51,7 \pm 2,81^{\mathrm{b}}$ & $36,82 \pm 1,63$ \\
Age reforme male (mois) & $30,8 \pm 4,45^{\mathrm{a}}$ & $23,9 \pm 1,46^{\mathrm{a}, \mathrm{b}}$ & $27,9 \pm 2,19^{\mathrm{a}}$ & $24,55 \pm 1,16$ \\
\hline
\end{tabular}

(a,b,c) les moyennes affectées de la même lettre sur la même ligne ne diffèrent pas significativement au seuil de 5\%

\subsection{Typologies des élevages}

4.4.1 Systèmes d'élevage: Le système d'élevage le plus pratiqué au niveau de l'ensemble des enquêtés est le type extensif $(53 \%)$. Cependant, au niveau des zones agro- écologiques, on constate des variations, avec la zone sahélienne qui présente environ $76 \%$ de ses éleveurs qui s'adonnent au système semi-intensif (Tableau 4).

Tableau 4: Proportion des types de système d'élevage pratiqués par zone agro-écologique.

\begin{tabular}{l|l|l}
\hline \multirow{2}{*}{ Zones agro-écologiques } & \multicolumn{2}{|c}{ Type de système (\%) } \\
\cline { 2 - 3 } & Système semi-intensif & Système extensif \\
\hline Sahélienne & 76 & 24 \\
Soudanienne & 48,78 & 51,22 \\
Soudano-sahélienne & 36,17 & 63,83 \\
\hline
\end{tabular}

\subsubsection{Typologie des éleveurs de poules} locales: L'Analyse des Correspondances Multiples (ACM) de 12 variables ont contribué à la représentation des individus dans l'espace bidimensionnel (Figure 3). Les 2 premières dimensions (axes) de la figure 3, expliquant $24,59 \%$ de la variation totale, ont permis une relative discrimination des éleveurs. 
Factor map

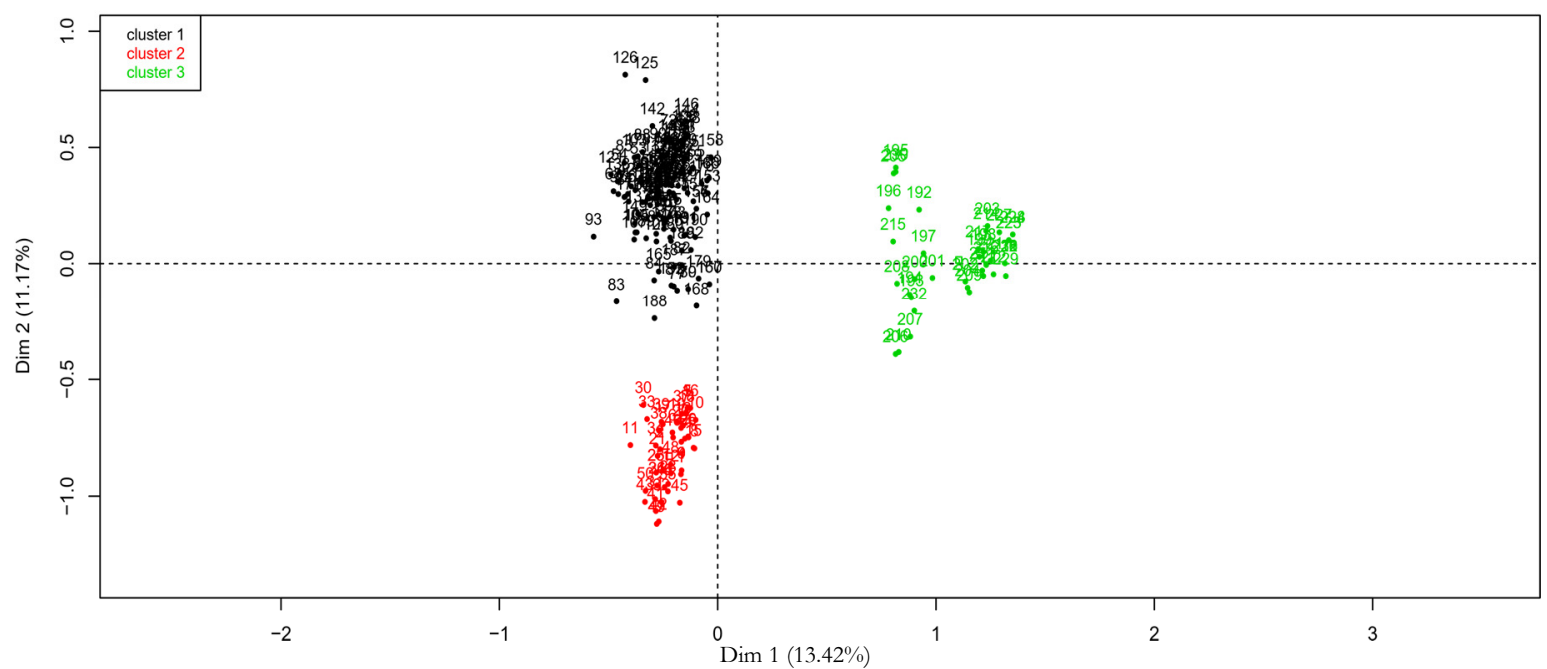

Figure 3 : Représentation graphique des groupes (clusters) sur les axes 1 et 2.

De l'analyse de la figure 3 il ressort que la classification hiérarchique a permis de distinguer 3 groupes (groupe 1 en noir, groupe 2 en rouge et groupe 3 en vert). Les pourcentages de la répartition des paramètres socio-économiques ont été calculés et présentés dans le Tableau 5.

Tableau 5: Répartition des paramètres socio-économiques par type d'éleveur en fonction de la significativité de la modalité.

\begin{tabular}{l|l|l|l}
\hline Variables & Type 1 & Type 2 & Type 3 \\
\hline soudan. Saheli & 100 & - & - \\
ethnie1 & 96,84 & - & 3,16 \\
objet1 & 73,12 & 26,88 & - \\
ethnie4 & 93,55 & - & - \\
syst2 & 73,17 & 9,76 & - \\
mort2 & 88,00 & - & - \\
effect3 & 75,00 & 2,08 & - \\
educat1 & 72,31 & - & - \\
origine2 & 77,14 & - & - \\
origine1 & 57,87 & 25,38 & - \\
educat0 & 50,53 & 32,63 & - \\
mort1 & 57,49 & - & 19,81 \\
syst1 & 46,79 & 34,86 & - \\
ethnie3 & 0,00 & - & 100 \\
ethnie2 & 24,69 & 61,73 & - \\
objet2 & - & - & 100 \\
soudan & - & - & 100 \\
saheli & - & 100 & - \\
expjeun & - & 31,82 & - \\
formatelev & - & 43,33 & - \\
hom & - & 16,56 & 20,86 \\
fem & - & 33,33 & 10,14 \\
jeune & - & - & 7,69 \\
expanc & - & 12,30 & - \\
format0 & - & - & 24,53 \\
effect2 & - & - & 11,11 \\
\hline
\end{tabular}


Sous type 1: Le sous type 1 est composé principalement des grands éleveurs de poules locales $(75 \%)$ ayant des effectifs supérieurs à 50 têtes et pouvant atteindre 2000 têtes. Il regroupe les éleveurs pratiquant plus le système extensif (73,17\%). Les mortalités sont essentiellement liées à la prédation (88\%). Le groupe se compose de la majorité $(73,12 \%)$ des éleveurs motivés par la recherche de revenus. Ce groupe est composé uniquement des éleveurs de la zone soudanosahélienne $(100 \%)$, d'ethnie essentiellement Mossi (96,84\%), Peulh (24,69\%) et regroupant la quasi-totalité des autres ethnies peu ou pas du tout représentées dans les autres zones que sont les Gourounsis, Tiefos, Bissas, Yaregas, Koursassis, Samos, Gourmantchés (93,55\%). Les éleveurs de poules locales, dans ce groupe, sont principalement des allogènes $(77,14 \%)$. Les éleveurs ne sachant ni lire, ni écrire $(50,53 \%)$ et ceux ayant été alphabétisés (72,31\%) sont présents dans ce groupe.

Sous type 2 : Le sous type 2 est dominé par les éleveurs pratiquant l'élevage semi-intensif $(34,86 \%)$, motivés par la recherche de revenus dans la pratique de l'élevage des poules locales. Ils ne savent ni lire, ni écrire avec pourtant une bonne proportion d'entre eux formée

\section{DISCUSSION}

La présente étude a montré que l'élevage des poules locales est une activité majoritairement masculine $(70,26 \%)$. Cette tendance masculine est conforme aux résultats obtenus au Tchad (Mopaté et al., 1998), en Inde (Moula et al., 2011), en Côte d'ivoire (Loukou, 2013), au Togo (Dao, 2015), et au Burkina Faso (Hien et al., 2005 ; Ouédraogo, 2018). Ce résultat est, par contre, contraire à celui obtenu par Gueye (1998), Branckaert (1999) et Fotsa et al.(2007) qui soutiennent que cette activité est traditionnellement féminine et pratiquée par environ $80 \%$ de femmes et des jeunes défavorisés dans la plupart des pays en développement. En milieu rural, la gestion patriarcale de la famille au Burkina Faso pourrait être à l'origine de ce pourcentage élevé de la gente masculine dans le secteur avicole spécifiquement en aviculture (43,33\% des éleveurs formés en la matière). Les éleveurs de ce groupe sont principalement des femmes ayant une petite expérience dans le domaine de l'aviculture en terme de durée dans l'activité. La totalité des éléments de ce groupe proviennent de la zone sahélienne, sont d'ethnie majoritairement Peulh (61,73\%) et sont pour la plupart des autochtones.

Sous type 3: Le groupe 3 est composé de la totalité $(100 \%)$ des éleveurs qui pratiquent l'élevage de poules locales pour des raisons de rite et de prestige avec des effectifs intermédiaires compris entre 26 et 50 têtes. Ces éleveurs n'ont généralement reçu aucune formation dans le domaine de l'élevage et sont principalement des jeunes hommes. La cause de la mortalité des poules dans ce groupe est principalement liée aux ravages faits par les maladies. La totalité des Bobos $(100 \%)$ se trouve dans ce groupe avec une faible proportion des Mossis (3,16\%). Les éleveurs de ce groupe sont tous de la zone soudanienne. En considérant les individus, 60,78\% des éleveurs enquêtés sont dans le groupe 1, 21,55\% dans le groupe 2 et $17,67 \%$ dans le groupe 3 .

traditionnel. Un constat similaire a été fait au Zimbabwe (Muchadeyi et al., 2004), en Côte d'Ivoire (Loukou, 2013) et au Congo (Moula et al., 2012). Néanmoins, l'immixtion des hommes dans la décision de vente des poules est déjà connue en Afrique. Elle est due à des considérations socio-culturelles qui donnent aux hommes ce droit de responsable et de gestionnaire de la famille au détriment des femmes (Gueye, 1998). Cette immixtion est illustrée ici par le faible pourcentage des femmes (29,74\%) qui se sont présentées personnellement à l'enquête, à cause de l'insistance des hommes à répondre au questionnaire à la place de leurs épouses, probablement pour leur éviter tout contact avec les visiteurs (Gueye, 1998). Cet état de fait limite la portée de l'élevage de poules en tant qu'outil ciblant la réduction de la pauvreté 
féminine. Ce type d'action de développement se doit donc de prendre en considération ces problématiques de genre en considération et s'insérer dans une logique plus globale de renforcement de la position de la femme dans la société rurale. L'objectif principal visé (motivation) pour l'élevage des poules locales est la recherche de revenus $(97,84 \%)$, comme rapporté par Pousga (2009). Toute chose qui justifie son utilisation pour l'éradication de la pauvreté et la promotion du genre (Guèye et al., 2000). Plus de la moitié des enquêtés n'appartient pas à une organisation professionnelle agricole (OPA) et compte une forte proportion d'illettrés $(40,95 \%)$. Ils sont diversement instruits et n'ont reçu aucune formation. Ce même constat a été fait par Fotsa et al.(2007) au Cameroun et Loukou (2013) en Côte d'Ivoire. Contrairement, à Loukou (2013), l'analphabétisme, n'est pas ici considéré comme une entrave à l'amélioration de l'aviculture familiale. Car, la plupart des éleveurs illettrés qui ont été interviewés font la prévention et les traitements contre les maladies aviaires dans le milieu rural avec les produits vétérinaires disponibles. Cette situation pouvant être expliquée par leur appartenance aux OPA, qui les font bénéficier des services fournis par cellesci. Les données indiquent que ce sont les petits éleveurs de poules locales (effectifs inférieurs à 25 têtes) qui sont les plus nombreux dans la zone d'étude. Ce résultat corrobore les observations faites par Loukou (2013) en Côte d'Ivoire, où les petits éleveurs de poules locales ont plutôt des effectifs inférieurs à 10 têtes. Cette différence de seuil pourrait s'expliquer par le fait que le Burkina Faso est un pays où l'élevage de poules locales est plus développé qu'en Côte d'Ivoire. La majorité des éleveurs de poules locales de la zone d'étude dispose de poulaillers/abris construits en banco et souvent de petites dimensions. D'autres auteurs comme Tadelle et Ogle (2001), El-Yuguda et al.(2007) et Ayssiwede et al.(2013) ont rapporté l'existence de ces mêmes types de poulaillers dans les élevages traditionnels en Ethiopie, au Nigéria et au Sénégal. Par ailleurs, comme l'a souligné ElYuguda et al.(2007), l'exiguïté des poulaillers conduit à l'entassement des fientes et à une rapide propagation des maladies. Ils constituent aussi des cachettes favorables pour, surtout, les ectoparasites, vu qu'ils sont rarement nettoyés et désinfectés. Par contre, contrairement à ces auteurs, les poulaillers de notre zone d'étude disposaient pour la plupart d'équipements d'élevage (abreuvoirs : 58,65\% et mangeoires : $73 \%$ ). Cette assez bonne adoption de ces équipements pourrait s'expliquer par les efforts consentis par l'Etat et ses partenaires au développement qui initient des micro-projets et subventions à l'endroit des éleveurs. Les systèmes d'élevage rencontrés dans la zone d'étude sont le système extensif et celui semiintensif, avec une prédominance du système extensif. La typologie définie dans la présente étude est proche de celle de Sonaiya et al.(1999), Alder (2005) et Ouattara (2015). En effet, ceuxci ont pu distinguer 2 types d'élevage traditionnel (extensif et amélioré) en fonction de leurs caractéristiques. Ces résultats confirment et complètent également ceux de Guèye (1998), Tadelle (2003) et Samson (2005) cité par Loukou (2013) qui ont rapporté dans leurs études qu'en milieu rural, les poules locales sont généralement élevées dans un système extensif. La présente étude indique cependant que dans la zone sahélienne, c'est le système semi-intensif qui est le plus prédominant avec environ $76 \%$ des éleveurs qui s'y adonnent. Cette particularité sahélienne pourrait s'expliquer par l'impact des projets et programmes de développement dans cette partie du pays. Tout comme au Cameroun (Fotsa, 2007), en Côte d'Ivoire (Loukou, 2013) et au Togo (Dao, 2015) les maladies, notamment la pseudo-peste aviaire couramment appelée maladie de Newcastle et la prédation constituent les principales causes de mortalités dans la zone d'étude. Cela s'expliquerait par une insuffisance de suivi sanitaire des élevages et l'inexistence ou la non-conformité des poulaillers. Des pratiques endogènes faisant appel à la pharmacopée traditionnelle ont été observées dans la zone d'étude et s'avèreraient efficaces contre certains parasitismes externes. Des pratiques similaires ont été rapportées par Fotsa (2007) au Cameroun. Notons cependant que la prédation 
ne semble pas constituer une contrainte majeure au Nigéria. En effet, El-Yuguda et al.(2007) ont déclaré que les maladies sont la seule cause de perte de poules locales. L'âge moyen d'entrée en ponte dans la zone d'étude $(6,57 \pm 010$ mois $)$ est similaire à celui rapporté par l'étude IEPC/MRA (2005) et conforme aux observations faites par Mahammi (2015) en Algérie, Akouango et al.(2004) à Brazzaville. Cet âge de la première ponte est, par contre, supérieur à celui observé en Ethiopie (Akouango et al., 2010) et inférieur à celui observé au Soudan (Yousif et Eltayeb, 2011) et au Congo (Moula, 2012). Les poules dans la zone d'étude pondent et couvent, en

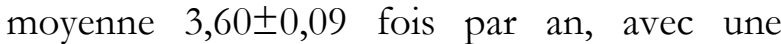
différence statistiquement significative, pour ce paramètre, entre les poules des zones soudanienne et sahélienne et celle de la zone soudano-sahélienne $(\mathrm{p}<0,05)$. Cette même valeur moyenne a été enregistrée pour les poules locales en Guinée (Missouhou et al., 1998), en Algérie (Mahammi, 2015) et au Sénégal (Mourad et al., 1997). La production annuelle d'œufs rapportée dans la littérature pour les poules locales varie entre 25 et 150 (Fotsa et al., 2010). Dans la présente étude elle est en moyenne de 41,33 $\pm 1,10$ œufs/an, donc dans cet intervalle.

\section{CONCLUSION}

La présente étude indique que l'élevage de poules locales est une activité généralement masculine et sa motivation première est la recherche de revenus. La caractérisation des système d'élevage de la poule locale a révélé l'existence de 2 types de systèmes. Il s'agit du type extensif et de celui semi-intensif. Le premier ne nécessite pratiquement pas d'apports (équipements, aliments et santé), tandis que le second exige une relative assistance en termes

\section{REMERCIEMENTS}

Les travaux ont été menés avec le soutien financier du Projet d'Appui au Développement du secteur de l'Elevage au Burkina (PADEL-B), le soutien technique du Laboratoire de Biologie et de Santé Animale (LaBioSA) de l'Institut de l'Environnement et de Recherches Agricoles
Par contre, elle reste supérieure à celle rapportée par l'étude IEPC/MRA (2005) et se situe à peu près à la moitié de celles observées en Algérie (Mahammi, 2014) et au Maroc (Benabdeljelil et Arfaoui, 2001). Elle se situe au tiers de la moyenne observée chez la poule locale Fayoum, la race Dandarawi en Egypte (Akouango et al., 2010) et chez la poule locale en Tunisie (Bessadok et al., 2003). Cette relative faible productivité de la poule locale, dans la zone d'étude, pourrait être expliquée par les conditions dans lesquelles elle est élevée. Des auteurs tels que Benabdeljalil et Bordas (2005), Fotsa (2008) et Akouango et al.(2010) indiquent que ces faibles performances pourraient être améliorées par la mise en place des plans de gestion et de sélection génétique ainsi que par l'amélioration des conditions d'élevage. L'âge moyen de la réforme chez la poule femelle est tardif et est de 36,82 $\pm 1,63$ mois. Il est également plus tardif chez les femelles que chez les mâles. Ces mêmes observations ont été rapportées par Ouédraogo (2018) et s'expliqueraient par le fait que les éleveurs dans la zone d'étude, s'inscrivent plus dans une dynamique d'élevage naisseur, d'où une certaine longévité des femelles dans les effectifs.

d'habitats, d'aliments variés et de soins à apporter par des professionnels de la santé animale. Des 3 groupes d'éleveurs identifiés, il ressort que ce sont plutôt les femmes qui pratiquent le système d'élevage de type semiintensif et se regrouperaient dans la zone sahélienne. Toutes choses pouvant expliquer une certaine précocité au niveau des oiseaux de cette zone.

(INERA) du Burkina Faso et de celui Laboratoire de Biologie Moléculaire et de Génétique (LABIOGENE) de l'Université Joseph Ki-ZERBO (UJKZ) de Ouagadougou. Les auteurs remercient l'ensemble des services 
techniques des sites de collecte et les éleveurs impliqués dans ce travail.

\section{RÉFÉRENCES}

Akounago F, Mouangou F. et Ganong G: 2004. Phénotypes et performances d'élevage chez des populations locales de volailles du genre Gallus gallus domesticus au Congo, Brazzaville, Cabier Agriculture, 13(3): 257-262.

Akouango F, Bandtaba P. et Ngokaka C: 2010. Croissance pondérale et productivité de la poule locale Gallus domesticus en élevage fermier au Congo, Animal Genetic Resources 46: 61-65.

Ayissiwede SB, Dieng A, Houinato MRB, Chrysostome CAAM, Issay I, Hornick JL. Et Missohou A: 2013. Elevage des poulets traditionnels ou indigènes au Sénégal et en Afrique Subsaharienne : état des lieux et contrainte 2011. Ann. Méd. Vét., 157: 103-119.

Benabdeljelil K, Arfaoui T. and Johnston P: 2001. Traditional poultry farming in Morocco. 10th International Conference of the Association of Institutions for Tropical Veterinary Medicine (AITVM) "Livestock, Community and environment" Copenhagen Denmark. 133-142 pp.

Benabdeljelil K. et Bordas A : 2005. Prise en compte des préférences des éleveurs pour la caractérisation des populations locales de poulets au Maroc. Sixièmes Journées de la Recherche Avicole, St Malo 30, 559p.

Bessadok A, Khochlf I. et El Gazzah M : 2003. Etat des ressources génétiques de la population locale du poulet en Tunisie. Tropicultura, 21(4) :167-172.

Branckaert RDS. and Guèye EF: 1999. FAO's programme for support to family poultry production. In: F. Dolberg et P.H. Petersen (eds) Poultry as tool in poverty eradication and promotion of gender equality. Actes d'un atelier, 22-26 Mars 1999, Tune Landboskole, Danemark. $244 \mathrm{p}$.
Cothenet G. et Bastianelli D: 1999. Les matières premières disponibles pour l'alimentation des volailles en zone humide. In production poulet de chair, paris: Edition ITAVI, 60-77 pp.

Dao B: 2015. Caractérisation phénotypique et moléculaire des populations des poules du Togo. Rapport technique, ITRA/MAEH, Togo : 73p

El-Yuguda AD, Ngulde IS, Abubakar MB. et Baba SS: 2007. Indices de santé, de conduite et de production des poulets villageoise dans des communautés rurales de l'Etat de Borno (Nigéria). Aviculture Familiale 17: 42-49.

Fotsa J, Rognon X, Tixier-Boichard M, Coquerelle G, Poné Kamdem D, Ngou Ngoupayou J, Manjeli Y. et Bordas A: 2010. Caractérisation phénotypique des populations de poules locales (Gallus Gallus) de la zone forestière dense humide à pluviométrie bimodale du Cameroun. Animal Genetic Resources 46 : 49-59.

Fotsa JC: 2008. Characterization of local chicken populations (Gallus gallus) in Cameroon. AgroParisTech, Institut des sciences et industries du vivant et de l'environnement UFR Génétique, Elevage et Reproduction. 301p.

Fotsa JC, Rognon X, Tixier-Boichard M, Ngou Ngoupayou JD, Poné Kamdem D, Manjeli Y. et Bordas A: 2007. Exploitation de la poule locale en zone de forêt humide du Cameroun. Bulletin de santé et production animale en Afrique, 55 : 59-73.

Guèye EF. and Dieng AS: 2000. Meat quality of indigenous and commercial chicken in Senegal. In : Sonaiya, E.B. (ed). Issues in family poultry Research and Development', in Proceeding of an 
International workshop held on. M'Bour, Senegal, 146-152 pp.

Guèye EF: 1998. Village egg and fowlmeat production in Africa-regional', Report World's Poultry Science Journal, 54: 73-86.

Hien OC, Diarra B, Drabo Y, Boly H. et Sawadogo L: 2005. Pratique de l'aviculture traditionnelle par les différents groupes ethniques de la région des cascades au Burkina Faso, Agronomie Africaine, 17 (3): 227-239.

IEPC/MRAH: 2005: Initiative « Elevage, pauvreté et croissance (IEPC)» Propositions pour un document national. Rapport principal. 142p.

Loukou NE: 2013. Caractérisation phénotypique et moléculaire des poulets locaux (Gallus gallus domesticus Linné, 1758) de deux zones agro-écologiques de la Côte-d'Ivoire. Thèse, Université Félix Houphouët-Boigny.

DOI: 10.13140/2.1.1686.5281. 205p.

Mahammi FZ, Gaouar SBS, Tabet-Aoul NT, Tixier-Boichard M. et SaïdiMehtar N: 2014. Caractéristiques morphobiométriques et systèmes d'élevage des poules locales en Algérie occidentale (Oranie). Cabier Agriculture 23 (6): 382392.

Mahammi FZ: 2015. Caractérisation phénotypique et moléculaire des populations de poule locale (Gallus gallus-domesticus). Thèse USTO, Oran, Algérie. 148p.

Missohou A, Sow R. et Ngwe-Assoumou C: 1998. Caractéristiques morphobiométriques de la poule du Sénégal. Animal Genetic Resources 24: 63-69.

Mopaté LY, Hendrikx P, Imadine M. et Idriss A: 1998. Socio-économie de la production aviaire dans la région du Nord-Guéra au centre du Tchad. Revue Scientifique $d u$ Tchad 5(2): 29 - 32.

Moula N, Detiffe N, Farnir F, AntoineMoussiaux N. et Leroy P: 2012. Aviculture familiale au Bas-Congo, République Démocratique du Congo (RDC). Livestock Research for Rural
Development, Volume 24 Article \# 74. Retrieved November 13, 2014, from http://www.lrrd.org/lrrd24/5/moul240 74.html

Mourad M, Bah AS. et Gbanamou G: 1997. Evaluation de la productivité et de la mortalité de la poule locale sur le plateau du Sankaran, Faranah, Guinée, entre 1993-1994. Rev. Elev.Med. Vet. Pays Trop., 50: 343-349.

MRA/PNDEL: 2010. Politique Nationale de Développement Durable de l'Elevage au Burkina Faso 2010-2025 (PNDEL). Burkina Faso. 45p.

MRAH/DGESS: 2018. Annuaire statistique 2016 du Ministère des Ressources Animales et Halieutiques 31_02_2019', Burkina Faso. 175p.

Muchadeyi FC, Sibanda S, Kusina NT, Kusina J. and Makuza S: 2004. The villager chicken production system in Rushinga Districy of Zimbabwe. Livestock Research for Rural Development. $\quad 16 \quad$ (67): 2004. http://www.lrrd.org//rrf16/6/much16 040.htm.

Ouattara S: 2015. Optimisation de l'utilisation des graines de Vigna unguiculata (L) Walp (niébé) comme source de protéines dans l'alimentation des volailles. Thèse de Doctorat unique en Développement Rural, Université Polytechnique de Bobo-Dioulasso, 173p.

Ouedraogo WR: 2018. Caractérisation socioéconomique, phénotypique zootechnique de la poule locale du Burkina Faso. Mémoire de fin de cycle d'ingénieur, Institut Polytechnique Rural de Formation et de Recherche Appliquée (IPR / IFRA) de Katibougou. 57p.

Pousga B: 2009. 'Synthèse des travaux den recherche en aviculture au Burkina-Faso, Rapport de recherche No 4.Réseau International pour le Développement de l'Aviculture Familiale, 18 (1/2) : 28-35.

Sonaiya EB, Branckaert RDS. and Gueye EF : 1999. Research and development option for family poultry. Adresse URL : http://www.fao. 
Org/ag/againfo/themes/fr/infpd/econ f_scope.

Tadelle D. and Ogle B: 2001. Village poultry production systems in the central Highlands of Ethiopia. Trop. Anim. Health Prod., 33: 521-537.

Yousif I. and Eltayeb N: 2011. Performance of Sudanese native dwarf and bare neck chicken raised under improved traditional production system. Agric. and Biol. J. North Ame 2: 860-866.

Zaman MA, Sorensen P. and Howlider MR: 2004. Egg production performances of a breed and three crossbreeds under semiscavenging system of management, Livestock Research for Rural Development, 16. Available at: http:/ /www.cipav.org.co/lrrd/lrrdl6/8 /zama16060.htm. 